\title{
Evaluación de la perfusión tisular en pacientes con choque séptico normodinámico versus hiperdinámico"
}

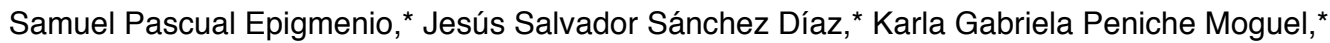 \\ Enrique Antonio Martínez Rodríguez, ${ }^{\ddagger}$ Josué Eli Villegas Domínguez, ${ }^{\S}$ María Verónica Calyeca Sánchez*
}

\section{RESUMEN}

Introducción: Desde que se documentaron las alteraciones del flujo sanguíneo a nivel microvascular en los pacientes con choque séptico, numerosos estudios a través de los años se han encargado de establecer el impacto pronóstico de dichas alteraciones en este grupo de pacientes.

Material y métodos: Se realizó un estudio de cohorte ambispectivo, longitudinal, descriptivo y analítico en pacientes con diagnóstico de choque séptico ingresados a la Unidad de Cuidados Intensivos (UCl) en el periodo comprendido entre el 15 de junio de 2015 y el 30 de julio de 2018. Los pacientes se clasificaron al ingreso en $\mathrm{SvcO}_{2} \geq 80 \%$ (choque séptico hiperdinámico) y $\mathrm{SvcO}_{2}<$ $80 \%$ (choque séptico normodinámico). Se utilizaron las variables de perfusión tisular lactato, $\Delta \mathrm{p}(\mathrm{v}-\mathrm{a}) \mathrm{CO}_{2}, \Delta \mathrm{p}(\mathrm{v}-\mathrm{a}) \mathrm{CO}_{2} / \Delta(\mathrm{a}-\mathrm{v}) \mathrm{O}_{2}$ y $\mathrm{ERO}_{2}$. Todos los análisis estadísticos se realizaron con el programa SPSSTM 22.0.

Resultados: En el periodo considerado, 82 pacientes cumplieron con los criterios de inclusión; de ellos, 60 (73.1\%) se clasificaron como estado de choque normodinámico y $22(26.9 \%)$ como hiperdinámico. El análisis multivariado reportó el ERO 2 con OR 20.373 (IC 95\%: 2.451-169.320; $p=0.005$ ), el lactato con OR 0.533 (IC 95\%: 0.146-1.948; $p=0.341$ ), el $\Delta(\mathrm{v}-\mathrm{a}) \mathrm{CO}_{2}$ con OR 4.848 (IC $95 \%$ : $1.201-19.565 ; \mathrm{p}=0.027$ ) y el $\Delta(\mathrm{v}-\mathrm{a}) \mathrm{CO}_{2} / \Delta(\mathrm{a}-\mathrm{v}) \mathrm{O}_{2}$ con OR $0.276($ IC $95 \%$ : $0.058-1.309 ; p=0.105$ )

Conclusión: Los pacientes con choque séptico presentan alteraciones de la perfusión tisular y la microcirculación; estas alteraciones no dependerán del estado hemodinámico (hiperdinamia o normodinamia) del paciente. Con una gasometría arterial y una gasometría venosa central podemos valorar la perfusión tisular y la microcirculación, midiendo variables como lactato, $\Delta \mathrm{p}(\mathrm{v}-\mathrm{a}) \mathrm{CO}_{2}, \Delta \mathrm{p}(\mathrm{v}-\mathrm{a}) \mathrm{CO}_{2} / \Delta(\mathrm{a}-\mathrm{v}) \mathrm{O}_{2}$ y $\mathrm{ERO}_{2}$ sin necesidad de dispositivos sofisticados.

Palabras clave: Choque séptico, normodinámico, hiperdinámico, perfusión tisular.

\section{SUMMARY}

Introduction: Since microvascular blood flow alterations were documented in patients with septic shock, numerous studies over the years have established the prognostic impact of these alterations in this group of patients.

Material and methods: A cohort, ambispective, longitudinal, descriptive and analytical study was performed in patients with a diagnosis of septic shock admitted to the Intensive Care Unit (ICU) in the period from June 15, 2015 to July 30, 2018. Patients were classified on admission in those with $\mathrm{SvcO}_{2} \geq$ $80 \%$ (hyperdynamic septic shock) and those with $\mathrm{SvcO}_{2}<80 \%$ (normodynamic septic shock). The following tissue perfusion variables were used: lactate, $\Delta p(v-a) C O_{2}, \Delta p(v-a) C_{2} / \Delta(a-v) O_{2}$ and $E_{2} O_{2}$. All statistical analyzes were done with the SPSSTM 22.0 program.

Results: In the period considered, 82 patients met the inclusion criteria, 60 of which $(73.1 \%)$ were classified as in normodynamic shock status and 22 (26.9\%) as hyperdynamic. The multivariate analysis reported the $E R O_{2}$ with OR 20.373 (95\% Cl: 2.451-169.320, $p=0.005$ ), the lactate with OR 0.533 (95\% Cl: 0.146-1.948, $p=0.341$ ), the $\Delta$ (va) $\mathrm{CO}_{2}$ with OR 4.848 (95\% Cl: 1.201 . $19.565, p=0.027$ ) and $\Delta(\mathrm{va}) \mathrm{CO} / \Delta(\mathrm{av}) \mathrm{O}_{2}$ with $\mathrm{OR} 0.276$ (95\% Cl: 0.058-1.309, $p=0.105)$

Conclusion: Patients with septic shock have alterations of tissue perfusion and microcirculation; these alterations will not depend on their hemodynamic state (hyperdynamia or normodynamia). With an arterial blood gas analysis and a central venous blood gas analysis, we can assess tissue perfusion and microcirculation, measuring variables such as lactate, $\Delta p(v-a) C_{2}, \Delta p(v-a) C_{2}$ $\triangle(a-v) \mathrm{O}_{2}$ and $E R \mathrm{O}_{2}$ without the need for sophisticated devices.

Key words: Septic shock, normodynamic, hyperdynamic, tissue perfusion.

\footnotetext{
" Ganador del Premio Académico «Mario Shapiro». Segundo lugar.

* Hospital de Especialidades Núm. 14, UMAE 189, IMSS, Veracruz.

¥ Centro Médico ABC, Ciudad de México.

$\S$ Universidad del Valle de México (UVM), Facultad de Medicina, Campus Veracruz.
}

Recepción: 21/08/2018. Aceptación: 20/09/2018.

Este artículo puede ser consultado en versión completa en http://www.medigraphic.com/medicinacritica
RESUMO

Introdução: Desde que foram documentadas as alterações do fluxo sanguíneo microvascular em pacientes com choque séptico, numerosos estudos ao longo dos anos têm sido responsáveis por estabelecer o impacto prognóstico dessas alterações nesse grupo de pacientes.

Material e métodos: Realizou-se um estudo de coorte, ambispectivo, longitudinal, descritivo e analítico em pacientes com diagnóstico de choque séptico internados na Unidade de Terapia Intensiva (UTI) no período de 15 de junho de 2015 a 30 de julho de 2018. Os pacientes foram classificados na admissão de acordo com $\mathrm{SvcO}_{2} \geq 80 \%$ (choque séptico hiperdinâmico) e $\mathrm{SvCO}_{2}<80 \%$ (choque séptico normodinâmico). Foram utilizadas as variáveis de perfusão tecidual: lactato, $\Delta p\left(v\right.$-a) $\mathrm{CO}_{2}, \Delta p(v-a) \mathrm{CO}_{2} / \Delta$ (a-v) $\mathrm{O}_{2}$ e $E R \mathrm{O}_{2}$. Todas as análises estatísticas foram realizadas com o programa SPSS 22.0. Resultados: No período considerado, 82 pacientes preencheram os critérios de inclusão, sendo 60 pacientes (73.1\%) classificados como estado de choque normodinâmico e 22 pacientes (26.9\%) como hiperdinâmicos. A análise multivariada relatou o ERO com OR 20.373 (95\% IC: $2.451-169.320, p=$ 0.005), o lactato com OR 0.533 (IC 95\%: 0.146-1.948, $p=0.341$ ), o $\Delta(v-a) \mathrm{CO}_{2}$ com OR 4.848 (IC 95\%: 1.201-19.565, p=0.027) e $\Delta(v-a) \mathrm{CO}_{2} / \Delta(a-v) \mathrm{O}_{2} \mathrm{com}$ OR 0.276 (IC 95\%: 0.058-1.309, $p=0.105$ ).

Conclusão: Os pacientes com choque séptico apresentam alterações de perfusão tecidual e microcirculação, sendo que estas não dependerão do estado hemodinâmico (hiperdinamia ou normodinamia) do paciente. Com uma gasometria arterial e uma gasometria venosa central, podemos avaliar a perfusão tecidual e microcirculação, medindo variáveis como: lactato, $\Delta p(v$ a) $\mathrm{CO}_{2}, \Delta p$ (v-a) $\mathrm{CO}_{2} / \Delta$ (a-v) $\mathrm{O}_{2}$ e $E R O_{2}$ sem a necessidade de dispositivos sofisticados.

Palavras-chave: Choque séptico, perfusão tissular, normodinâmico, hiperdinâmico.

\section{INTRODUCCIÓN}

No existe mayor estrés para el cuerpo humano que el choque séptico. Un cúmulo de acontecimientos neuroendocrinos y humorales ocurrirán con la finalidad de mantener el equilibrio. Desde 1920, Aubb mencionaba que la respuesta del organismo será del tamaño de la lesión. Hoy en día, consideramos al choque séptico como una «reacción sistémica exagerada del huésped a la infección». 1,2 Esta respuesta exagerada causa disfunción orgánica aguda y pone en peligro la vida, alcanzando una mortalidad hospitalaria del 18 al 35\%. Alrededor del mundo, 19 millones de personas presentarán sepsis cada año; de ellas, 14 millones sobrevivirán con incapacidad a largo plazo y deterioro de la calidad de vida por la morbilidad. Solo la mitad de los pacientes que padecieron sepsis tendrá una recuperación completa; $40 \%$ de los afectados retomará su vida laboral. ${ }^{3}$ Todo lo anterior convierte a la sepsis en un problema de salud pública mundial y obliga al intensivista a redoblar esfuerzos para «sobrevivir a la sepsis».

Poco más de 25 años son los que llevamos hablando de sepsis: desde 1992, con el primer consenso del American College of Chest Physicians/Society of Critical Care Medicine,${ }^{4}$ que estableció el concepto de «síndrome de respuesta inflamatoria sistémica», además, se definió sepsis, sepsis severa, choque séptico y, por 
otro lado, síndrome de disfunción orgánica múltiple. En el año 2001, el grupo de expertos de la Society of Critical Care Medicine (SCCM), la European Society of Intensive Care Medicine (ESICM), la American College of Chest Physicians (ACCP), la American Thoracic Society (ATS) y la Surgical Infection Society (SIS) mantuvieron dichos conceptos y definiciones, pero agregaron nuevos parámetros generales, inflamatorios, hemodinámicos y de perfusión tisular. ${ }^{5}$ En fechas recientes, el grupo de trabajo Sepsis Definitions Task Force ha publicado definiciones actualizadas de sepsis y choque séptico. Es de resaltar que en este consenso desapareció el concepto de sepsis severa y se agregó el quick SOFA (qSOFA) como una herramienta para el diagnóstico temprano del paciente con sepsis. ${ }^{6}$

Desde que De Backer y sus colaboradores ${ }^{7}$ documentaron las alteraciones del flujo sanguíneo a nivel microvascular en los pacientes con choque séptico, numerosos estudios a través de los años se han encargado de establecer el impacto pronóstico de dichas alteraciones en este grupo de pacientes. ${ }^{8}$ Hoy en día, diferentes métodos están disponibles para detectar hipoperfusión tisular y alteración microvascular en el paciente críticamente enfermo, que van desde la clínica (hipotensión, piel moteada, acrocianosis, llenado capilar lento o disminución de la temperatura) hasta otros más sofisticados, como la tonometría gástrica, capnometría sublingual, espectroscopia o near infrared spectroscopy (NIRS), orthogonal polarization spectral imaging (OPS) y sidestream dark field imaging (SDF). ${ }^{9}$ Por otro lado, la hiperlactatemia, el incremento de la $\Delta \mathrm{p}(\mathrm{v}-\mathrm{a}) \mathrm{CO}_{2}$ (diferencia venoarterial de dióxido de carbono) o el aumento de la $\Delta p(v-a)$ $\mathrm{CO} 2 / \Delta(\mathrm{a}-\mathrm{v}) \mathrm{O}_{2}$ (diferencia venoarterial de dióxido de carbono/diferencia arteriovenosa de oxígeno) en pacientes con choque séptico se relacionan con hipoperfusión tisular y alteración de la microcirculación, pues estas variables han sido correlacionadas con alteraciones microcirculatorias a nivel sublingual en diferentes estudios. ${ }^{10,11}$

La correcta relación entre macrocirculación y microcirculación (bien llamada "coherencia hemodinámica») debe ser el objetivo de todas las maniobras realizadas en el paciente con choque séptico. Corregir los problemas sistémicos o variables macrohemodinámicas debería conducir a la mejoría en la perfusión tisular y la microcirculación. Por desgracia, lo anterior no sucede en el paciente con choque séptico, siendo la «incoherencia hemodinámica» una de sus principales características. ${ }^{12}$ La falta de coherencia hemodinámica conduce a hipoperfusión tisular y variaciones microcirculatorias, alterando la relación $\mathrm{DO}_{2} / \mathrm{VO}_{2}$ (disponibilidad y consumo de oxígeno) y llegando finalmente a la disoxia celular, caracterizada por metabolismo anaerobio persistente, disfunción orgánica múltiple y muerte. ${ }^{13}$

Cuando hablamos de microcirculación nos referimos a los vasos con diámetro menor de $100 \mu \mathrm{m}$, lo que incluye arteriolas, metarteriolas, capilares y vénulas. Debemos entender que la microcirculación es un sistema de distribución funcional del flujo sanguíneo; por lo tanto, se encarga de mantener un adecuado balance entre el aporte y el consumo de sustancias, sobre todo oxígeno, entre los tejidos y la sangre. Serán las propiedades reológicas de los fluidos -en este caso, de la sangre - las que se encarguen de la adecuada perfusión tisular y, a su vez, la correcta microcirculación. Además, los componentes convectivo y difusivo resultan esenciales para el adecuado transporte de oxígeno. Los cambios en la microcirculación que presenta el paciente con choque séptico son secundarios a la inflamación, activación de la coagulación, activación del complemento y el daño endotelial. ${ }^{14}$ Estas alteraciones microvasculares comprenden disminución en la proporción de vasos perfundidos, la densidad capilar y la velocidad del flujo, junto con mayor heterogeneidad. Las anomalías de la perfusión tisular y microvasculares en el paciente críticamente enfermo están presentes con mayor frecuencia en los no sobrevivientes respecto a los sobrevivientes. Por otra parte, sabemos bien que estas anomalías pueden ser predictores independientes de morbimortalidad en los pacientes con choque séptico. En consecuencia, la adecuada evaluación de la perfusión tisular y la microcirculación abre el panorama respecto a la fisiopatología y tratamiento de la sepsis. ${ }^{15}$

La microcirculación es un componente clave del sistema cardiovascular. De hecho, en general, el paciente con choque séptico exhibe gasto cardiaco normal o alto; dicho en otras palabras, estado de choque normodinámico o hiperdinámico. Por lo tanto, el choque séptico es considerado un estado de «choque microcirculatorio", condición definida por la presencia de hipoperfusión tisular a pesar de flujo sanguíneo regional y sistémico normal. ${ }^{16,17}$ La definición de hiperdinámico va más allá de la condición clínica del paciente con choque séptico, pues en el sentido estricto debería incluir índice cardiaco $\geq 4.0 \mathrm{~L} / \mathrm{min} / \mathrm{m}^{2}$ o saturación venosa central de oxígeno $\left(\mathrm{SvcO}_{2}\right) \geq \mathrm{a} 80 \%$. Esta última resulta útil para valorar el metabolismo global de oxígeno. Los niveles bajos de la $\mathrm{SvcO}_{2}$ reflejan bajo gasto cardiaco, extracción excesiva de oxígeno, bajos niveles de hemoglobina y/o bajo nivel de presión arterial oxígeno. Por el contrario, niveles elevados de $\mathrm{SvcO}_{2}$ pueden significar aporte $\mathrm{o}$ disponibilidad de oxígeno $\left(\mathrm{DO}_{2}=\right.$ contenido arterial de oxígeno $x$ gasto cardiaco x 10) muy alto, disminución del consumo celular de oxígeno (disfunción mitocondrial) y/o, en raras ocasiones, un gran cortocircuito arteriovenoso. ${ }^{18,19}$ La perfusión tisular y la microcirculación tienen un comportamiento independiente de la hemodinamia sistémica; determinar si difieren en el choque normodinámico versus el choque hiperdinámico es el propósito de este trabajo, así como si estas diferencias influyen en los resultados. 
Justificación: El choque séptico es un gran problema de salud pública: afecta a millones de personas alrededor del mundo, con una morbimortalidad que permanece elevada. Representa la principal causa de choque en las unidades de cuidados intensivos (UCl). La correcta evaluación de los pacientes con choque séptico desde su ingreso nos permitirá ubicar a aquellos pacientes con mayor posibilidad de beneficiarse con el tratamiento. Igual que otras patologías, la identificación temprana y una adecuada clasificación y tratamiento en las horas iniciales al diagnóstico mejorarán los resultados. Las guías actuales para el manejo del paciente con choque séptico recomiendan la evaluación de marcadores de la macrocirculación, pero también la valoración de la perfusión tisular y la microcirculación representan un objetivo terapéutico prometedor.

Objetivo: Comparar las variables de perfusión tisular en los pacientes con choque séptico normodinámico versus hiperdinámico.

Hipótesis: Existen diferencias entre las variables de perfusión tisular en los pacientes con choque séptico normodinámico versus hiperdinámico al ingreso a la unidad de cuidados intensivos.

\section{MATERIAL Y MÉTODOS}

Se autorizó por el Comité de Investigación y Ética del hospital. No se realizó consentimiento informado debido a que se trató de un estudio de riesgo mínimo y no intervención.

Tipo de estudio: Estudio de cohorte ambispectivo, longitudinal, descriptivo y analítico.

Universo de trabajo y lugar de desarrollo: Pacientes con diagnóstico de choque séptico (según las guías de la campaña Sobreviviendo a la Sepsis del año 2016) ingresados a la Unidad de Cuidados Intensivos en el periodo comprendido del 15 de junio de 2015 al 30 de julio de 2018.

Criterios de inclusión: Pacientes que ingresaron a la Unidad de Cuidados Intensivos con diagnóstico de choque séptico según las guías de la campaña Sobreviviendo a la Sepsis del año 2016. Pacientes con catéter venoso central (posición correcta corroborada por radiografía de tórax). Pacientes con gasometría arterial y venosa al ingreso a la Unidad de Cuidados Intensivos. Pacientes mayores de 18 años.

Criterios de exclusión: Pacientes con disfunción del catéter venoso central. Registro de variables incompleto. Pacientes con fístula arteriovenosa.

Criterios de eliminación: Pacientes embarazadas. Pacientes con orden de no reanimación o de máximo alcance terapéutico. Pacientes con choque séptico que finalizaron su tratamiento en otra unidad médica. Pacientes que iniciaron su tratamiento en nuestra $\mathrm{UCl}$ y solicitaron su traslado a otra unidad médica para continuar el manejo.

\section{Metodología}

Al ingreso a la Unidad de Cuidados Intensivos, en todo paciente que cumplió con los criterios de inclusión se obtuvieron gasometrías arterial y venosa central en un mismo tiempo y se procesaron en equipo GEM Premier 3000; se tomaron en ese momento muestras de sangre para medir las variables necesarias para el estudio. Los pacientes se clasificaron de acuerdo con el $\mathrm{SvcO}_{2}$ : si fue $\geq$ de $80 \%$, se ingresaron al grupo de choque séptico hiperdinámico; si fue < de $80 \%$, se ingresaron al grupo de choque séptico normodinámico. Fueron considerados sobrevivientes al ser dados de alta de la Unidad de Cuidados Intensivos o al llegar al día 30 de estancia. Se realizó el vaciado de datos en una hoja de Excel para posteriormente analizar las variables con el programa SPPSTM 22.

\section{Análisis estadístico}

Los datos obtenidos se presentan como medidas de tendencia central y dispersión. Las variables de estudio cuantitativas con distribución normal se analizaron con t Student y las cualitativas con $\chi^{2}$. En las variables con distribución anormal se utilizó prueba exacta de Fisher y U Mann-Whitney según el caso; tuvo significancia estadística un valor de $\mathrm{p}<0.05$. El análisis multivariado usó los puntos de corte ya establecidos en la literatura para las variables de perfusión tisular: lactato, $\Delta \mathrm{p}(\mathrm{v}-\mathrm{a}) \mathrm{CO}_{2}$, $\Delta \mathrm{p}(\mathrm{v}-\mathrm{a}) \mathrm{CO}_{2} / \Delta(\mathrm{a}-\mathrm{v}) \mathrm{O}_{2}$ y extracción de oxígeno $\left(\mathrm{ERO}_{2}\right)$. Se empleó razón de momios (odds ratio, OR) para evaluar los valores de corte como riesgos relativos para mortalidad de acuerdo a la $\mathrm{SvcO}_{2}$. Todos los análisis estadísticos se realizaron con el programa SPSSTM 22.0.

\section{RESULTADOS}

En el periodo considerado, 82 pacientes cumplieron con los criterios de inclusión; de ellos, 60 (73.1\%) se clasificaron como estado de choque normodinámico $\left(\mathrm{SvcO}_{2}\right.$ $<80 \%)$ y $22(26.9 \%)$ como hiperdinámico $\left(\mathrm{SvcO}_{2} \geq\right.$ $80 \%)$. No se observó diferencia significativa en la edad promedio de ambos grupos (normodinámico: $54.72 \pm$ 18.93; hiperdinámico: $54.59 \pm 15.86 ; p=0.9$ ). Del total de pacientes, $45.6 \%$ (39) correspondieron al género masculino. EI SAPS II, los días de estancia en la UCI y los días de VM (ventilación mecánica) no presentaron significancia estadística, con $p=0.3, p=0.5, p=0.9$, respectivamente. El sitio de infección más frecuente fue el abdominal, con $46.3 \%$ de los casos, seguido del pulmonar, con $24.3 \%$. El Servicio de Urgencias contribuyó con $37 \%$ de los ingresos a la UCl, a la par con el Servicio de Cirugía General (37\%). De los pacientes con choque séptico normodinámico, $45 \%$ (27 pacientes) fallecieron, mientras que en el grupo de choque séptico 
Tabla 1: Características basales de la población.

\begin{tabular}{lccc}
\hline Variable & $\begin{array}{c}\text { Normodinámico } \\
\mathrm{n}=60\end{array}$ & $\begin{array}{c}\text { Hiperdinámico } \\
\mathrm{n}=22\end{array}$ & $\mathrm{p}$ \\
\hline Edad (media/DE) & $54.72 \pm 18.93$ & $54.59 \pm 15.86$ & 0.9 \\
Género $(\mathrm{H})(\%)$ & $23(52.3)$ & $16(42.1)$ & 0.4 \\
SAPS II (media/DE) & $68.80 \pm 13.47$ & $66.00 \pm 12.22$ & 0.3 \\
Días de estancia en la UCl & $6.38 \pm 6.54$ & $5.45 \pm 3.10$ & 0.5 \\
(media/DE) & & & \\
Días de VM (media/rango) & $4(21)$ & $4(12)$ & 0.9 \\
Norepinefrina ( $\mu \mathrm{g} / \mathrm{kg} / \mathrm{min})$ & $0.31( \pm 0.19)$ & $0.25( \pm 0.15)$ & 0.2 \\
TRRLC (\%) & $30(50.0)$ & $7(31.8)$ & 0.1 \\
Mortalidad (total) $(\%)$ & $27(45.0)$ & $11(50.0)$ & 0.8 \\
\hline
\end{tabular}

Abreviaturas: $\mathrm{DE}=$ desviación estándar, $\mathrm{H}=$ hombre; SAPS $\|=$ Simplified Acute Physiology Score; $\mathrm{UCl}=$ Unidad de Cuidados Intensivos; $\mathrm{VM}=$ ventilación mecánica; TRRLC = terapia de reemplazo renal lenta continua.

Fuente: Directa.
Tabla 2: Análisis bivariado de las variables de perfusión tisular.

\begin{tabular}{lccr}
\hline Variable & $\begin{array}{c}\text { Normodinámico } \\
n=60\end{array}$ & $\begin{array}{c}\text { Hiperdinámico } \\
n=22\end{array}$ & \multicolumn{1}{c}{$\mathrm{p}$} \\
\hline $\mathrm{SvcO}_{2}(\%)($ media/DE) & $65.97 \pm 7.73$ & $83.23 \pm 3.22$ & $<0.001$ \\
$\mathrm{ERO}_{2}(\%)($ media/DE) & $32.84 \pm 8.50$ & $17.43 \pm 5.32$ & $<0.001$ \\
$\mathrm{Lactato} \mathrm{mmol} / \mathrm{L}$ & $4.88 \pm 3.29$ & $3.66 \pm 2.76$ & 0.100 \\
$\Delta(\mathrm{v}-\mathrm{a}) \mathrm{CO}_{2} \mathrm{mmHg}($ media/DE) & $6.17 \pm 3.44$ & $4.77 \pm 3.49$ & 0.100 \\
$\Delta \mathrm{p}(\mathrm{v}-\mathrm{a}) \mathrm{CO}_{2} / \Delta(\mathrm{a}-\mathrm{v}) \mathrm{O}_{2} \mathrm{mmHg} /$ & $1.65 \pm 1.19$ & $2.43 \pm 1.86$ & 0.020 \\
$\mathrm{~mL}(\mathrm{media} / \mathrm{DE})$ & & & \\
Base mEq/L (media/DE) & $-9.96 \pm 6.87$ & $-10.68 \pm 6.31$ & 0.600 \\
Brecha aniónica corregida & $17.99 \pm 5.12$ & $20.41 \pm 5.64$ & 0.060 \\
$\mathrm{mEq} / \mathrm{L}$ (media/DE) & & & \\
\hline
\end{tabular}

Abreviaturas: $\mathrm{DE}=$ desviación estándar, $\mathrm{SvCO}_{2}=$ saturación venosa central de oxígeno, $\mathrm{ERO}_{2}=$ extracción de oxígeno; $\Delta(\mathrm{v}-\mathrm{a}) \mathrm{CO}_{2}=$ diferencia venoarterial de dióxido de carbono, $\Delta(\mathrm{v}-\mathrm{a}) \mathrm{CO}_{2} / \Delta \Delta(\mathrm{a}-\mathrm{v}) \mathrm{O}_{2}=$ índice entre la diferencia venoarterial de dióxido de carbono y la diferencia arteriovenosa de oxígeno.

Fuente: Directa.

Tabla 3: Análisis multivariado de las variables de perfusión tisular como factores de riesgo para mortalidad.

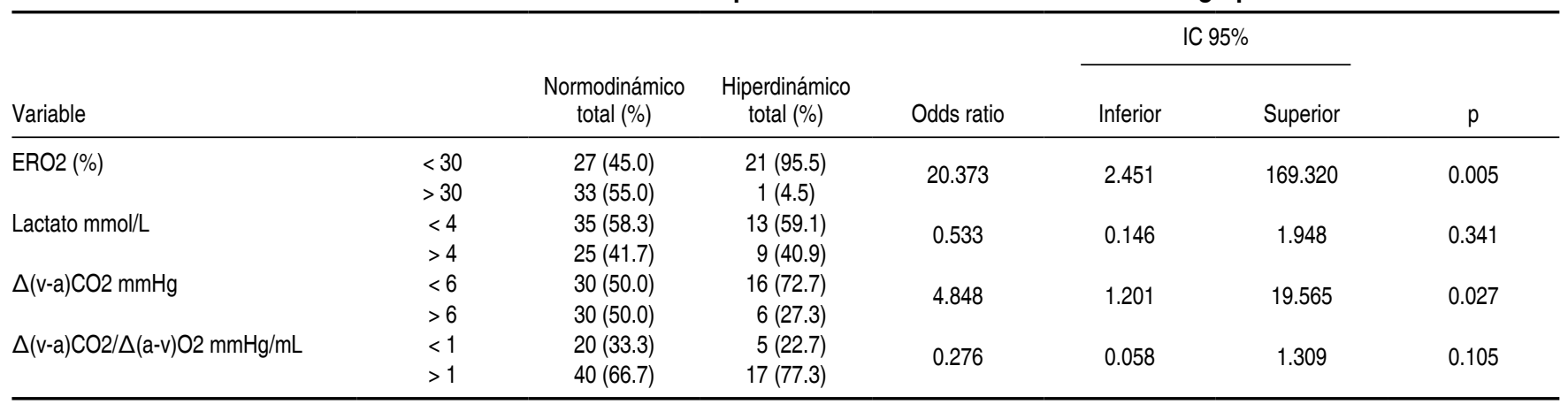

Abreviaturas: $\mathrm{ERO}_{2}$ : extracción de oxígeno; $\Delta(\mathrm{v}-\mathrm{a}) \mathrm{CO}_{2}=$ diferencia venoarterial de dióxido de carbono; $\Delta(\mathrm{v}-\mathrm{a}) \mathrm{CO}_{2} / \Delta(\mathrm{a}-\mathrm{v}) \mathrm{O}_{2}=$ índice entre la diferencia venoarterial de dióxido de carbono y la diferencia arteriovenosa de oxígeno.

Fuente: Directa.

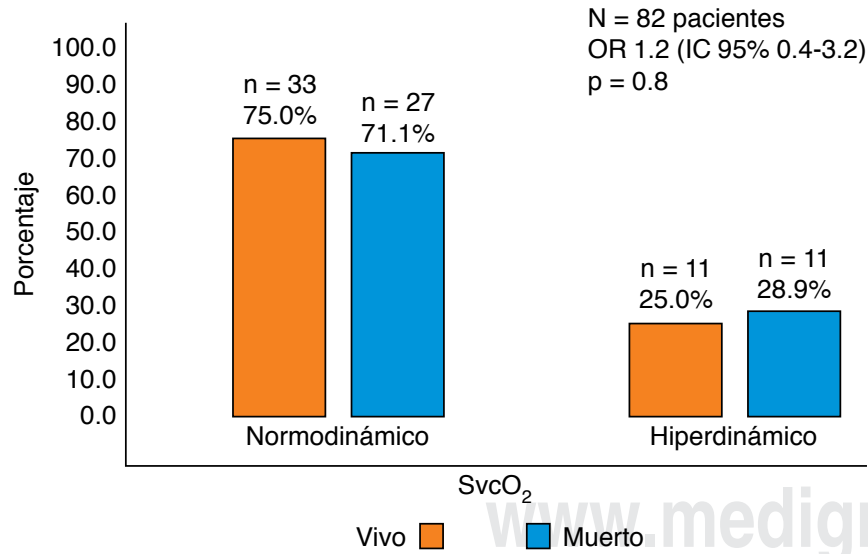

Abreviaturas: $\mathrm{SvcO}_{2}=$ saturación venosa central de oxígeno.

Figura 1: Saturación venosa central de oxígeno al ingreso a la UCl como factor pronóstico de mortalidad.

hiperdinámico, $50 \%$ (11 pacientes), esto con una $p=$ 0.8 . El resto de las características generales de los pacientes se detallan en la Tabla 1.

Las variables que se utilizaron para evaluar la perfusión tisular al ingreso a la UCI por medio del análisis bivariado fueron el $\mathrm{ERO}_{2}$, lactato, $\Delta \mathrm{p}(\mathrm{v}-\mathrm{a}) \mathrm{CO}_{2}$ y $\Delta \mathrm{p}(\mathrm{v}-\mathrm{a})$ $\mathrm{CO}_{2} / \Delta(\mathrm{a}-\mathrm{v}) \mathrm{O}_{2}$. De ellas, el $\mathrm{ERO}_{2}$ en el grupo normodinámico fue de $32.84 \pm 8.50$ y en el grupo hiperdinámico de $17.43 \pm 5.32$, con un valor de $p \leq 0.001$. El valor del lactato fue $4.88 \pm 3.29$ y $3.66 \pm 2.76$ (normodinámico e hiperdinámico, respectivamente), con $p=0.1$. El valor del $\Delta p(v-a) \mathrm{CO}_{2}$ fue de $6.17 \pm 3.44$ y $4.77 \pm 3.49$ (normodinámico e hiperdinámico, respectivamente), sin diferencia estadística, con $p=0.1$. Por otro lado, el $\Delta \mathrm{p}(\mathrm{v}-\mathrm{a}) \mathrm{CO}_{2} / \Delta(\mathrm{a}-\mathrm{v}) \mathrm{O}_{2}$ fue de $1.65 \pm 1.19$ para el grupo normodinámico versus $2.43 \pm 1.86$ para el hiperdinámico, con una $p=0.02$. El resto del análisis bivariado se puede ver en la Tabla 2.

En el análisis multivariado, las variables como factor de riesgo para mortalidad en ambos grupos fueron el $\mathrm{ERO}_{2}$-con OR 20.373 (IC 95\%: 2.451-169.320; $\mathrm{p}=$ 0.005 ) - , el lactato - con OR 0.533 (IC 95\%: 0.146$1.948 ; p=0.341)-$, el $\Delta(v-a) \mathrm{CO}_{2}-$ con OR 4.848 (IC 95\%: $1.201-19.565 ; \mathrm{p}=0.027)-\mathrm{y}$ el $\Delta(\mathrm{v}-\mathrm{a}) \mathrm{CO}_{2} / \Delta(\mathrm{a}-\mathrm{v})$ $\mathrm{O}_{2}$-con OR 0.276 (IC 95\%: 0.058-1.309; $\mathrm{p}=0.105$ )(Tabla 3). Al clasificar a los pacientes con choque séptico de acuerdo al desenlace final, la $\mathrm{SvcO}_{2}$ al ingreso a la $\mathrm{UCI}$ no fue un factor pronóstico para el mismo (Figura 1). 


\section{DISCUSIÓN}

Los niveles de $\mathrm{SvcO}_{2}$ reflejan alteraciones en la extracción de oxígeno $\left(\mathrm{ERO}_{2}\right)$ en pacientes con choque séptico. La $\mathrm{ERO}_{2}$ también resulta útil para determinar el estado hemodinámico de estos pacientes: un incremento $(>30 \%)$ sugiere hipodinamia o metabolismo celular elevado; por otro lado, la disminución (<20\%) se asocia con hiperdinamia o metabolismo celular bajo. En ambas situaciones, la perfusión tisular o la microcirculación pueden ser deficientes, siendo la falta de coherencia hemodinámica (macrocirculación $\neq$ microcirculación) una de las características en estos enfermos. ${ }^{20}$ La clasificación de normodinámico versus hiperdinámico fue realizada con la $\mathrm{SvcO}_{2} \geq 80 \%$, pues esta condición, que va más allá de un estado clínico, también la observamos en nuestro análisis bivariado, donde se documentó $\mathrm{ERO}_{2}$ de 32.84 versus 17.43 para el grupo de normodinamia e hiperdinamia, respectivamente, con significancia estadística $(<0.001)$. Estudios han correlacionado niveles superiores al $80 \%$ de $\mathrm{SvcO}_{2}$ con mortalidad en pacientes que presentan choque séptico. $\mathrm{La} \mathrm{SvcO}_{2}$ fue de $85 \%$ (78 a 89) en los no sobrevivientes, en comparación con $79 \%$ (72 a 87 ) en los sobrevivientes $(p=0.009){ }^{21}$ Los niveles de $\mathrm{SvcO}_{2}$ (altos o bajos) pueden reflejar la gravedad del estado de choque, representando el porcentaje de oxígeno que la célula no utiliza (por alteraciones del metabolismo celular) de la sangre arterial. Esto podría deberse a mala distribución de la perfusión tisular por trastornos microcirculatorios que impiden la llegada del oxígeno a los tejidos. Una cohorte retrospectiva que incluyó 619 pacientes evaluó la mortalidad en los pacientes con choque séptico según los niveles de $\mathrm{SvcO}_{2}$ alcanzados tras la reanimación; la mortalidad fue de $40 \%$ (IC 95\% 29-53\%), 21\% (IC 95\% 17-25\%) y $34 \%$ (IC 95\% 25-44\%), respectivamente, según los grupos: $<70 \%, 71-89 \%$ y $\geq 90 \% .{ }^{22}$ Nuestro estudio incluyó 82 pacientes (60 normodinámicos y 22 hiperdinámicos), sin encontrar diferencias en la mortalidad: $27(45 \%)$ versus $11(50 \%)$, respectivamente, con $p=0.8$.

Por medio de la evaluación detallada de una gasometría arterial y una gasometría venosa central podemos realizar un análisis profundo no solo del estado hemodinámico del paciente con choque séptico, sino también valorar la perfusión tisular y la microcirculación a través de la medición de variables como lactato, $\Delta \mathrm{p}(\mathrm{v}-\mathrm{a}) \mathrm{CO}_{2}, \Delta \mathrm{p}(\mathrm{v}-\mathrm{a}) \mathrm{CO}_{2} / \Delta(\mathrm{a}-\mathrm{v}) \mathrm{O}_{2}$ y $\mathrm{ERO}_{2}$. Lo anterior nos resulta útil en el contexto de choque microcirculatorio, definido por la presencia de tejido hipoperfundido a pesar de flujo sanguíneo sistémico y regional normal, condición distintiva del choque séptico. ${ }^{23}$ Las alteraciones de la perfusión tisular y de la microcirculación fueron documentadas en ambos grupos de pacientes (normodinámicos e hiperdinámicos) con las variables mencionadas, las cuales en su mayoría rebasan los puntos de corte: lactato $>4 \mathrm{mmol} / \mathrm{L}, \Delta \mathrm{p}(\mathrm{v}-\mathrm{a}) \mathrm{CO}_{2}>6$ $\mathrm{mmHg}$ y $\Delta \mathrm{p}(\mathrm{v}-\mathrm{a}) \mathrm{CO}_{2} / \Delta(\mathrm{a}-\mathrm{v}) \mathrm{O}_{2}>1 \mathrm{mmHg} / \mathrm{mL}$.

Hoy en día existe suficiente evidencia científica que asocia el metabolismo anaerobio con niveles elevados de lactato; esta es la razón de su utilidad como herramienta diagnóstica y pronóstica en pacientes con choque séptico. No debemos olvidar que el incremento puede ocurrir en una gran variedad de escenarios clínicos en ausencia de metabolismo anaerobio. ${ }^{24-27}$ Las alteraciones de la perfusión tisular o de la microcirculación en pacientes con choque séptico se asocian a valores de lactato superiores a $4 \mathrm{mmol} / \mathrm{L}$, con una sensibilidad de $69 \%$ y especificidad de $77 \% .{ }^{28}$ El análisis bivariado de nuestro estudio no muestra diferencias significativas en ambos grupos respecto a los niveles de lactato, con $4.88 \mathrm{mmol} / \mathrm{L}$ y $3.66 \mathrm{mmol} / \mathrm{L}$ para el grupo normodinámico versus el hiperdinámico, respectivamente, con una $p=0.1$. Esta tendencia se mantiene en el análisis multivariado, con OR 0.533 (IC 95\% 0.1461.948, $p=0.34)$.

La $\triangle \mathrm{p}(\mathrm{v}-\mathrm{a}) \mathrm{CO}_{2}$ es la diferencia que existe en los niveles de $\mathrm{pCO}_{2}$ (presión de dióxido de carbono) entre la sangre venosa central y la sangre arterial; en condiciones normales, su valor es de dos a seis $\mathrm{mmHg}^{29}$ Frente al incremento en la producción de $\mathrm{CO}_{2}$ (dióxido de carbono) a nivel tisular en condiciones de metabolismo anaerobio, los niveles de $\mathrm{CO}_{2}$ en la sangre venosa central serán mayores que los de la sangre arterial, resultando en una $\Delta \mathrm{p}(\mathrm{v}-\mathrm{a}) \mathrm{CO}_{2}$ aumentada; bajo este sustento se afirma que constituye un reflejo de flujo sanguíneo inadecuado, mala perfusión tisular o alteraciones de la microcirculación. ${ }^{30,31}$ Es importante mencionar que el principal determinante del flujo sanguíneo tisular es el gasto cardiaco (GC); está bien documentado que el incremento ( $>6 \mathrm{mmHg}$ ) de la $\Delta \mathrm{p}(\mathrm{v}-\mathrm{a}) \mathrm{CO}_{2}$ se da en forma paralela a la disminución del GC. ${ }^{32} \mathrm{El}$ análisis multivariado muestra diferencia significativa entre el grupo de normodinámicos e hiperdinámicos, con OR 4.848 (IC 95\% 1.201-19.565, p = 0.027), estableciendo como punto de corte $6 \mathrm{mmHg}$. Una $\Delta \mathrm{p}(\mathrm{v}-\mathrm{a})$ $\mathrm{CO}_{2}>6 \mathrm{mmHg}$ en el grupo de normodinámicos puede ser explicada, como ya mencionamos, por disminución del GC (hipoxia isquémica) o incremento de la producción de $\mathrm{CO}_{2}$ (hipoxia tisular o hipoxia hipóxi$\mathrm{ca}$,) situación que no se diferenció en nuestro estudio. Para el grupo de hiperdinámicos, la $\Delta \mathrm{p}(\mathrm{v}-\mathrm{a}) \mathrm{CO}_{2}<6$ $\mathrm{mmHg}$ puede estar relacionada con el adecuado $\mathrm{GC}$ de este grupo por la hiperdinamia per se. Estos números nos podrían hacer pensar mejor perfusión tisular y microcirculación en el grupo hiperdinámico, aunque no necesariamente, tomando en cuenta los factores de confusión comentados (gasto cardiaco versus metabolismo anaerobio). La $\triangle \mathrm{p}(\mathrm{v}-\mathrm{a}) \mathrm{CO}_{2}$ (ABC 0.684, IC 95\% 
0.568-0.799, sensibilidad 90\%, especificidad $41 \%$ ) goza de mejor área bajo la curva $(A B C)$ que lactato, $\mathrm{SvcO}_{2}$, pero no que la diferencia $\Delta \mathrm{p}(\mathrm{v}-\mathrm{a}) \mathrm{CO}_{2} / \Delta(\mathrm{a}-\mathrm{v}) \mathrm{O}_{2}$ (ABC 0.721, IC 95\% 0.612-0.813, sensibilidad 94\%, especificidad 48\%); por esto, la consideraremos el mejor marcador de alteraciones de la perfusión tisular o microcirculación. ${ }^{33}$

Existe buena correlación entre $\Delta \mathrm{p}(\mathrm{v}-\mathrm{a}) \mathrm{CO}_{2} / \Delta(\mathrm{a}-\mathrm{v})$ $\mathrm{O}_{2}$ y cociente respiratorio (CR), que es la relación entre el $\mathrm{CO}_{2}$ producido $\left(\mathrm{VCO}_{2}\right)$ y el $\mathrm{O}_{2}$ consumido $\left(\mathrm{VO}_{2}\right)$ : $\mathrm{CR}=\mathrm{VCO}_{2} / \mathrm{VO}_{2} \cdot{ }^{34} \mathrm{Es}$ el indicador más confiable de la presencia de metabolismo anaerobio en pacientes críticamente enfermos; por ende, de mala perfusión tisular y alteraciones de la microcirculación. ${ }^{35}$ Sus valores de referencia son variables en diferentes literaturas, aunque podemos tomar un punto de corte $\leq 1 \mathrm{mmHg} /$ $\mathrm{mL}$. Mesquida y sus colaboradores ${ }^{36}$ demostraron que la presencia de valores persistentemente elevados de $\Delta \mathrm{p}(\mathrm{v}-\mathrm{a}) \mathrm{CO}_{2} / \Delta(\mathrm{a}-\mathrm{v}) \mathrm{O}_{2}$ se asocia con mayor mortalidad en pacientes con choque séptico. Nuestro análisis bivariado muestra una media $>1 \mathrm{mmHg} / \mathrm{mL}$ en ambos grupos, con $1.65 \mathrm{mmHg} / \mathrm{mL}$ para el grupo normodinámico versus $2.43 \mathrm{mmHg} / \mathrm{mL}$ para el hiperdinámico, con una $p$ $=0.02$. Lo anterior deja claro que en ambos grupos existen alteraciones de la perfusión tisular y la microcirculación, que pudiera encontrarse aún más alterada en el grupo hiperdinámico, aunque el análisis multivariado no apoya esta tendencia, OR 0.276 (IC 95\% 0.058-1.309, $p=0.105)$. Edul y su grupo ${ }^{37}$ realizaron la evaluación cuantitativa de la microcirculación sublingual en pacientes con choque séptico hiperdinámico con el dispositivo sidestream dark field imaging (SDF) y demostraron perfusión tisular reducida, disminución de la velocidad de flujo y cambios en la densidad vascular. A través de este trabajo documentamos que las alteraciones de la perfusión tisular y la microcirculación de los pacientes con choque séptico no difieren en presencia de hiperdinamia o normodinamia, y que para su diagnóstico no es indispensable utilizar dispositivos sofisticados (NIRS, OPS o SDF), pues la correcta interpretación de variables como lactato, $\Delta \mathrm{p}(\mathrm{v}-\mathrm{a}) \mathrm{CO}_{2}$ y $\Delta \mathrm{p}(\mathrm{v}-\mathrm{a}) \mathrm{CO}_{2} / \Delta(\mathrm{a}-\mathrm{v}) \mathrm{O}_{2}$ puede ser suficiente.

\section{CONCLUSIÓN}

Los pacientes con choque séptico presentan alteraciones de la perfusión tisular y la microcirculación; estas no dependen de su estado hemodinámico (hiperdinamia o normodinamia). Con una gasometría arterial y una gasometría venosa central podemos valorar la perfusión tisular y la microcirculación en pacientes con choque séptico, midiendo variables como lactato, $\triangle \mathrm{p}(\mathrm{v}-\mathrm{a}) \mathrm{CO}_{2}$, $\Delta \mathrm{p}(\mathrm{v}-\mathrm{a}) \mathrm{CO}_{2} / \Delta(\mathrm{a}-\mathrm{v}) \mathrm{O}_{2}$ y $\mathrm{ERO}_{2}$ sin necesidad de dispositivos sofisticados. Lo anterior resulta útil en el contexto de choque microcirculatorio.

\section{BIBLIOGRAFÍA}

1. Reuter DA, Russell JA, Mekontso Dessap A. Beta-blockers in septic shock to optimize hemodynamics? Yes. Intensive Care Med. 2016;42(10):1607-1609.

2. Weissman $\mathrm{C}$. The metabolic response to stress: an overview and update. Anesthesiology. 1990;73:308-327.

3. Prescott HC, Angus DC. Enhancing recovery from sepsis: a review. JAMA. 2018;319(1):62-75.

4. American College of Chest Physicians/Society of Critical Care Medicine Consensus Conference: definitions for sepsis and organ failure and guidelines for the use of innovative therapies in sepsis. Crit Care Med. 1992;20(6):864-874.

5. Levy MM, Fink MO, Marshall JC, Abraham E, Angus D, Cook D, et al. 2001 SCCM/ESICM/ACCP/ATS/SIS International Sepsis Definitions Conference. Intensive Care Med. 2003;29(4):530538.

6. Singer M, Deutschman CS, Seymour CW, Shankar-Hari M, Annane $\mathrm{D}$, Bauer $\mathrm{M}$, et al. The third international consensus definitions for sepsis and septic shock (sepsis-3). JAMA. 2016;315(8):801-810.

7. De Backer D, Creteur J, Preiser JC, Dubois MJ, Vincent JL. Microvascular blood flow is altered in patients with sepsis. $\mathrm{Am} \mathrm{J}$ Respir Crit Care Med. 2002;166:98-104.

8. Hernández G, Teboul JL. Is the macrocirculation dissociated from the microcirculation in septic shock? Intensive Care Med. 2016;42(10):1621-1624.

9. Rivera-Solís G, Sánchez-Díaz JS, Martínez-Rodríguez EA García-Méndez RC, Huanca-Pacaje JM, Calyeca-Sánchez MV. Clasificación clínica de la perfusión tisular en pacientes con choque séptico basada en la saturación venosa central de oxígeno (SvcO2) y la diferencia venoarterial de dióxido de carbono entre el contenido arteriovenoso de oxígeno $(\Delta \mathrm{P}(\mathrm{v}-\mathrm{a})$ $\left.\mathrm{CO}_{2} / \mathrm{C}(\mathrm{a}-\mathrm{v}) \mathrm{O}_{2}\right)$. Med Crit. 2016;30(5):283-289.

10. Hernandez G, Boerma EC, Dubin A, Bruhn A, Koopmans M, Edul VK, et al. Severe abnormalities in microvascular perfused vessel density are associated to organ dysfunctions and mortality and can be predicted by hyperlactatemia and norepinephrine requirements in septic shock patients. J Crit Care. 2013;28:538. e9-538.e14.

11. Ospina-Tascón GA, Umaña M, Bermúdez WF, Bautista-Rincón DF, Valencia JD, Madriñán HJ. ¿Can venous-to-arterial carbon dioxide differences reflect microcirculatory alterations in patients with septic shock? Intensive Care Med. 2016;42:211-221.

12. Ince $\mathrm{C}$. Hemodynamic coherence and the rationale for monitoring the microcirculation. Crit Care. 2015;19 Suppl 3:S8.

13. Bakker J, Gris P, Coffernils M, Kahn RJ, Vincent JL. Serial blood lactate levels can predict the development of multiple organ failure following septic shock. Am J Surg. 1996;171:221-226.

14. Tafner PF, Chen FK, Rabello RF, Corrêa TD, Chaves RC, Serpa AN. Recent advances in bedside microcirculation assessment in critically ill patients. Rev Bras Ter Intensiva. 2017;29(2):238-247.

15. De Backer D, Donadello K, Sakr Y, Ospina-Tascon G, Salgado $D$, Scolletta $S$, et al. Microcirculatory alterations in patients with severe sepsis: impact of time of assessment and relationship with outcome. Crit Care Med. 2013;41:791-799.

16. Ince $\mathrm{C}$. The microcirculation is the motor of sepsis. Crit Care. 2005;9:S13-S19.

17. Gutierrez $\mathrm{G}$. The rate of oxygen release and its effect on capillary $\mathrm{O} 2$ tension: a mathematical analysis. Respir Physiol. 1986;63:79-96.

18. Valenzuela-Sánchez F, Bohollo de Austria R, Monge-García I, Gil-Cano A. Shock séptico. Med Intensiva. 2005;29:192-200.

19. Marx G, Reinhart K. Venous oximetry. Curr Opin Crit Care. 2006;12:263-268.

20. Gattinoni L, Pesenti A, Matthay M. Understanding blood gas analysis. Intensive Care Med. 2018;44(1):91-93.

21. Textoris J, Fouché L, Wiramus S, Antonini F, Tho S, Martin C, et al. High central venous oxygen saturation in the latter stages of septic shock is associated with increased mortality. Crit Care. 2011;15(4):R176.

22. Pope JV, Jones AE, Gaieski DF, Arnold RC, Trzeciak S, Shapiro NI. Multicenter study of central venous oxygen saturation 
$(\mathrm{ScvO}(2))$ as a predictor of mortality in patients with sepsis. Ann Emerg Med. 2010;55:40-46.

23. Kanoore Edul VS, Ince C, Dubin A. What is microcirculatory shock? Curr Opin Crit Care. 2015;21:245-252.

24. Mikkelsen ME, Miltiades AN, Gaieski DF, Goyal M, Fuchs BD, Shah CV, et al. Serum lactate is associated with mortality in severe sepsis independent of organ failure and shock. Crit Care Med. 2009;37:1670-1677.

25. Nguyen HB, Rivers EP, Knoblich BP, Jacobsen G, Muzzin A, Ressler JA, et al. Early lactate clearance is associated with improved outcome in severe sepsis and septic shock. Crit Care Med. 2004;32:1637-1642.

26. Jansen TC, van Bommel J, Schoonderbeek FJ, Sleeswijk Visser SJ, van der Klooster JM, Lima AP, et al. Early lactate-guided therapy in intensive care unit patients: a multicenter, open label, randomized controlled trial. Am J Respir Crit Care Med. 2010;182:752-776.

27. Levy B. Lactate and shock state: the metabolic view. Curr Opin Crit Care. 2006;12(4):315-332.

28. Mallat J, Lemyze M, Meddour M, Pepy F, Gasan G, Barrailler S, et al. Ratios of central venous-to-arterial carbon dioxide content or tension to arteriovenous oxygen content are better markers of global anaerobic metabolism than lactate in septic shock patients. Ann Intensive Care. 2016;6(1):10.

29. Groenveld $A B$. Interpreting the venous-arterial $\mathrm{pCO}_{2}$ difference. Crit Care Med. 1998;26:979-980.

30. Lamia B, Monet X, Teboul JL. Meaning of arterio-venous $\mathrm{pCO}_{2}$ difference in circulatory shock. Minerva Anestesiol. 2006;72:597604.

31. Van der Linden P, Rausin I, Deltell A, Bekrar Y, Gilbart E, Bakker $J$, et al. Detection of tissue hypoxia by arteriovenous gradient for $\mathrm{pCO}_{2}$ and $\mathrm{pH}$ in anesthetized dogs during progressive hemorrhage. Anesth Analg. 1995;80:269-275.

32. Cuschieri J, Rivers EP, Donnino MW, Katilius M, Jacobsen G, Nguyen HB, et al. Central venous-arterial carbon dioxide difference as an indicator of cardiac index. Intensive Care Med. 2005;31:818-822.

33. He HW, Liu DW, Long Y, Wang XT. High central venous-toarterial $\mathrm{CO}_{2}$ difference/arterial-central venous $\mathrm{O}_{2}$ difference ratio is associated with poor lactate clearance in septic patients after resuscitation. J Crit Care. 2016;31(1):76-81.

34. Mekontso-Dessap A, Castelain V, Anguel N, Bahloul M, Schauvliege F, Richard C, et al. Combination of venoarterial $\mathrm{PCO} 2$ difference with arteriovenous $\mathrm{O} 2$ content difference to detect anaerobic metabolism in patients. Intensive Care Med. 2002;28:272-277.

35. Monnet X, Julien F, Ait-Hamou N, Lequoy M, Gosset C, Jozwiak $\mathrm{M}$, et al. Lactate and venoarterial carbon dioxide difference/ arterial-venous oxygen difference ratio, but not central venous oxygen saturation, predict increase in oxygen consumption in fluid responders. Crit Care Med. 2013;41:1412-1420.

36. Mesquida J, Saludes P, Gruartmoner G, Espinal C, Torrents $E$, Baigorri $F$, et al. Central venous-to-arterial carbon dioxide difference combined with arterial-to-venous oxygen content difference is associated with lactate evolution in the hemodynamic resuscitation process in early septic shock. Crit Care. 2015;19:126.

37. Edul VS, Ince C, Vazquez AR, Rubatto PN, Espinoza ED, Welsh $S$, et al. Similar microcirculatory alterations in patients with normodynamic and hyperdynamic septic shock. Ann Am Thorac Soc. 2016;13(2):240-247.

Conflicto de intereses: Los autores reportan que no hay conflicto de intereses. No se requirió financiamiento externo.

\author{
Correspondencia: \\ Dr. Jesús Salvador Sánchez Díaz \\ Av. Cuauhtémoc s/n, \\ Formando Hogar, 91897, \\ Veracruz, Ver. \\ Teléfono fijo: (229) 9343500 \\ Teléfono celular: 5538105375 \\ E-mail: drsalvadorsanchezdiaz@gmail.com
}

